PALABRAS CLAVE

Hogares

Ingresos familiares

Bienestar social

Programas de acción

Evaluación de programas

Modelos econométricos

Datos estadísticos

Uruguay

\section{Gabriel Burdín}

Investigador del Instituto de Economía,

Universidad de la República,

Uruguay

œ gburdin@iecon.ccee.edu.uy

Gioia de Melo

Investigadora del Instituto de Economía,

Universidad de la República,

Uruguay

• gioiademelo@iecon.ccee.edu.uy
REVISTA CEPAL $98 \cdot$ AGOSTO 2009

\section{Prestaciones sociales}

en Uruguay:

¿por qué hay beneficiarios que no las solicitan?

\author{
Gabriel Burdin y Gioia de Melo
}

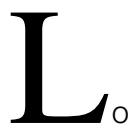

os programas de transferencias monetarias han cobrado gran importancia en América Latina. La preocupación por su adecuada focalización se ha centrado en excluir a las personas que no cumplen los requisitos de elegibilidad. En cambio, se ha prestado menos atención al hecho de que ellos no alcanzan a la totalidad de su población objetivo, en parte debido a que un conjunto de personas ni siquiera solicita los beneficios. En el presente artículo se analizan los factores determinantes de la no solicitud de prestaciones sociales.

El caso estudiado es el Plan de Atención Nacional a la Emergencia Social, un programa de transferencia de ingresos implementado en el Uruguay entre 2005 y 2007. Se cuantificó que más de la quinta parte de los hogares elegibles no se inscribieron en el programa. Las causas determinantes de la decisión de postular se estimaron mediante un modelo probit. Las evidencias obtenidas son ampliamente compatibles con los estudios teóricos y empíricos sobre el tema. 


\section{I}

\section{Introducción}

¿Por qué motivo los hogares que satisfacen los requisitos de elegibilidad para recibir determinadas prestaciones sociales no las solicitan? Esta falta de respuesta por parte de los posibles beneficiarios es lo que en los estudios sobre evaluación de los programas sociales se ha denominado el problema de non take-up. El objetivo del presente documento es ofrecer algunas explicaciones preliminares a esta pregunta, considerando el caso del Plan de Atención Nacional a la Emergencia Social (PANES) aplicado en el Uruguay.

Hernanz, Malherbert y Pellizzari (2004) señalan que hay al menos tres razones que justifican el estudio de la no solicitud de prestaciones sociales. En primer lugar, el hecho de que un programa social alcance solo a una fracción de su población objetivo reduce su eficacia respecto del propósito original. Segundo, un mejor conocimiento de los principales factores que influyen en la determinación de postular permite estimar con más exactitud las consecuencias de un cambio de las políticas en materia de costo fiscal, así como mejorar su diseño. Tercero, si la decisión de no solicitar el beneficio es parcialmente involuntaria - por ejemplo, obedece a mala información, problemas administrativos o procedimientos estigmatizadores - se producen disparidades en el tratamiento de los individuos, pese a que el sistema de protección social debería ser igual para todos. En otras palabras, algunas personas tendrían dificultades para ejercer determinados derechos sociales.

La mayoría de los estudios disponibles sobre el non take-up se ha realizado en los países de la Organización de Cooperación y Desarrollo Económicos (OCDE) y especialmente en Inglaterra y los Estados Unidos, que tienen una tradición más amplia en materia de programas sociales focalizados. ${ }^{1}$ Sin embargo, el tema ha adquirido una importancia creciente en los países de América Latina debido a la implementación de numerosos programas selectivos de transferencias monetarias. ${ }^{2}$ Para elegir a

\footnotetext{
${ }^{1}$ La mayoría de las investigaciones sobre el non take-up se ha realizado en Inglaterra (Atkinson, 1989; Craig, 1991; Corden, 1995, y Currie, 2004, entre otros) y los Estados Unidos (Ashenfelter, 1983; Moffitt, 1983; Blank and Ruggles, 1996; Anderson y Meyer, 1997; Bollinger y David, 2001), si bien en la última década el tema ha cobrado relevancia en los países europeos.

${ }^{2}$ Cabe citar los que se han aplicado en México (Progresa-Oportunidades), Colombia (Familias en Acción), Honduras (Programa de Asignación
}

los beneficiarios, se utilizan mecanismos basados en los recursos y la situación socioeconómica de los hogares (proxy means tests) que, inevitablemente, conducen a errores de focalización (Cornia y Stewart, 1995). Estos pueden ser de dos tipos y suponen la inclusión de personas que no se encuentran dentro de la población objetivo, pero también la no inclusión de otras que sí cumplen los requisitos de elegibilidad de los programas. Como lo señalan Coady, Grosh y Hoddinott (2004), se ha hecho hincapié en minimizar los primeros — perfeccionando las fórmulas estadísticas para identificar a los participantes que no reúnen los requisitos pertinentes-, pero se ha subestimado la importancia de los segundos, lo que en la práctica implica que un segmento de la población más vulnerable no accede a las prestaciones sociales.

En este sentido, disponer de nuevas evidencias sobre los factores que determinan la decisión de inscribirse podría ser de utilidad para esclarecer algunos aspectos problemáticos del diseño de programas sociales focalizados. Ello reviste especial importancia para el Uruguay, dado que el tema no se ha abordado en otros estudios. Tampoco se ha tratado mayormente en las evaluaciones de los programas sociales realizadas en la región. Asimismo, la necesidad de mejorar la comprensión de este fenómeno obedece al hecho de que el instrumento de focalización empleado en el PANES ha sido utilizado - con algunas adaptaciones- en otros programas de implementación reciente tales como el nuevo régimen legal de asignaciones familiares, la iniciativa Objetivo Empleo y la ley de asistencia a la vejez.

Por último, se entiende que contar con un modelo explicativo de la decisión de los hogares o personas de postular a los programas sociales puede ser un recurso importante para los estudios de evaluación ex ante de las políticas, basados en microsimulaciones. El objetivo de este tipo de trabajos, que han tenido creciente desarrollo

Familiar), Nicaragua (Red de Protección Social), Bolivia (proyecto Beca Futuro), Ecuador (Bono de desarrollo humano), Chile (Subsidio unitario familiar) y Brasil (Programa de Erradicación del Trabajo Infantil (PETI), Bolsa Escola, Bolsa Familia). En general, estos programas tienen un doble objetivo: por una parte, operar como sostén de ingreso ante situaciones de privación extrema en el acceso a los recursos; por otra, promover la acumulación de capital humano, particularmente a nivel de los miembros más jóvenes de los hogares, mediante la exigencia de contrapartidas en materia de alimentación, controles sanitarios y asistencia a centros educacionales. Véase CEPAL (2006). 
a nivel nacional y regional, es analizar la magnitud y el signo de las consecuencias de distintas alternativas de política en algunas variables de interés - normalmente la indigencia, la pobreza y la distribución del ingreso-, previo a su implementación. Sin embargo, ellos adolecen de la limitación de que por lo general suponen la focalización perfecta de los programas, lo que puede conducir a una medición inapropiada de los efectos de las políticas. En este sentido, la posibilidad de conocer las decisiones de los hogares puede contribuir a calibrar en forma más precisa los resultados.

En el presente estudio se estima un modelo probit de la probabilidad de postular al Plan Nacional de Atención a la Emergencia Social, a los efectos de identificar los principales factores que explican esta decisión. El modelo se especifica buscando aproximarse a las causas más importantes señaladas en los trabajos teóricos y empíricos: factores monetarios, costos de información y costos sociales y psicológicos. Los datos utilizados corresponden a la Encuesta Nacional de Hogares Ampliada 2006 y la Encuesta Continua de Hogares 2007.

El documento está organizado de la siguiente manera. Después de la Introducción, en la sección II se define más concretamente el problema de investigación a abordar. En la sección III se discuten los estudios teóricos sobre los factores determinantes de la decisión de postular a los programas sociales y se revisan los antecedentes de los trabajos empíricos. En la sección IV se especifica el modelo de análisis y en la siguiente se resumen las características del PANES. En la sección VI se detallan las fuentes de información utilizadas, se identifica la magnitud relativa del grupo de hogares de interés y se presentan las estadísticas descriptivas. En la sección VII se describen los resultados de las estimaciones y, por último, se realizan los comentarios finales.

\section{II}

\section{Definición del problema de investigación}

Van Oorschot (1996) identificó tres aproximaciones posibles al análisis del problema de no solicitud de prestaciones sociales o non take-up. Dicha tipología permite establecer con mayor exactitud el objeto de estudio del presente documento.

a) Non take-up primario o secundario. La no solicitud primaria implica que las personas elegibles no solicitan la prestación de que se trate, mientras que la secundaria ocurre cuando los individuos elegibles que sí lo hacen son rechazados por los administradores del programa.

b) Non take-up parcial o total. Esta modalidad se refiere a la situación en que una persona solicita una prestación determinada, pero recibe tan solo parte de ella. ${ }^{3}$ Esto puede ocurrir tanto porque el

\footnotetext{
${ }^{3}$ En Europa existen programas en que las prestaciones varían en función de la diferencia entre el ingreso del hogar y la línea de pobreza. En este sentido, es posible que debido a un error administrativo o de focalización, un solicitante aceptado en uno de ellos reciba un monto de dinero distinto al que se requeriría para equiparar el ingreso del hogar con la línea de pobreza.
}

postulante entregó información inexacta como por un error de e) valuación de la administración del programa.

c) Non take-up permanente o transitoria. Es posible que las personas elegibles requieran de un cierto período de tiempo para tomar conocimiento de la existencia del programa, considerarse elegibles, solicitar la prestación y finalmente recibirla, lo cual determina una situación de no solicitud transitoria.

En este marco, el presente estudio se centra en los factores determinantes del non take-up primario y total a los programas sociales. No se aborda el problema de no solicitud secundario vinculado a los errores o conducta discrecional de la administración del programa en cuanto a la aplicación del mecanismo de focalización para elegir a los beneficiarios.

Por otra parte, el non take-up parcial no parece ser relevante en el caso del PANES, puesto que este consistió de una transferencia monetaria uniforme. Finalmente, si bien se considera importante hacer una distinción entre la falta de respuesta permanente y transitoria, para abordarla se requeriría disponer de datos de panel que permitieran observar el comportamiento de los individuos elegibles a lo largo del tiempo. 


\section{III}

\section{Revisión del material bibliográfico teórico y empírico}

Los factores determinantes de la no solicitud de prestaciones sociales generalmente se modelan como una relación entre los beneficios y los costos de postular, haciendo hincapié en la relevancia de los costos directos e indirectos de inscribirse en este tipo de programas. Ello suele ser costoso para los hogares, puesto que implica trasladarse hasta las oficinas públicas, tramitar la documentación requerida, llenar formularios y hacer una declaración de ingresos, entre otros. En este sentido, se supone que los hogares decidirán presentarse en la medida en que el monto del beneficio y la duración del período de otorgamiento compensen con creces los costos incurridos, incluidos los de carácter no pecuniario.

De acuerdo con Fuchs (2007), los factores que influyen en la decisión de postular a los programas sociales pueden clasificarse en cuatro grandes grupos.

a) Factores monetarios. Cuanto más elevado sea el beneficio en relación al ingreso del hogar, mayor será el incentivo a solicitarlo. En caso de que este varíe según las características del postulante, se prevé que cuanto más significativo sea el monto que cada hogar estima le corresponde, mayor será el incentivo a solicitar la prestación. Las expectativas sobre la situación económica futura son extremadamente importantes en lo que refiere a la decisión de inscribirse en los programas sociales. Si una persona tiene pocas esperanzas de superar su situación de carencia por sus propios medios, el período de tiempo en que espera recibir el beneficio será más largo y por lo tanto amerita hacer la solicitud del caso. Sin embargo, existen costos pecuniarios en materia de transporte, tramitación de los documentos necesarios y otros que pueden ser valorados a la hora de evaluar la posibilidad de postular al programa.

b) Costos de procesamiento de la información relativa a los programas sociales y complejidad de los procedimientos de postulación. Ello supone diversos grados de falta de información, hasta un límite máximo de ni siquiera saber que existe un beneficio determinado. El conocimiento que se tenga del programa puede afectar la percepción sobre la probabilidad de recibir la prestación del caso, así como las expectativas de recibirla en función del costo de solicitarla.

c) Costos de espera vinculados a la duración del proceso de solicitud y a la incertidumbre respecto de los resultados del mismo.

d) Costos sociales y psicológicos. En los países occidentales, las normas sociales indican que las personas deberían sustentarse por sí mismas. En este sentido, el hecho de solicitar una prestación social puede concebirse como una desviación de las reglas de trabajo y un símbolo de fracaso. Según Lindbeck, Nyberg y Weibull (1999), el estigma que genera el hecho de recibir una prestación podría definirse como la sanción a que da lugar la violación de la norma social en virtud de la cual las personas deben autosustentarse con su propio trabajo. Como lo señala Elster (1989), la violación de las normas sociales desencadena intensas emociones negativas, tanto por parte de quien no las cumple como de otras personas.

En localidades pequeñas donde los contactos son más personalizados y en que por lo tanto las acciones individuales son fácilmente observables, es posible que el temor a las sanciones sociales contribuya a desincentivar la postulación a programas sociales. No obstante, aun en contextos más impersonales como el que caracteriza a las grandes ciudades, la interiorización de las normas a nivel individual podría generar un efecto similar. En otras palabras, una persona se abstendrá de solicitar una prestación si considera que ello representa un fracaso que afecta su autoestima (Moffitt, 1983; Atkinson, 1995; Sen, 1995). Asimismo, los individuos podrían tomar la decisión de no solicitar un beneficio debido a la pérdida de integridad que implica tener que someterse a una evaluación de elegibilidad (Mood, 2005).

Por otra parte, las interacciones sociales y, en general, los efectos del entorno son especialmente significativos en la medida en que afectan los costos de información y de estigma. En diversos estudios se ha documentado la importancia de la interacción social y en particular del comportamiento del grupo de pares (peer group effects) en materia de pobreza. Si las 
restricciones o sanciones vinculadas a determinadas conductas son recíprocas entre las personas, el costo individual que enfrenta cada una de ellas dependerá de cuán difundidos se encuentren dichos comportamientos en el resto del grupo (Durlauf, 2002). En este sentido, la propensión a solicitar un beneficio puede verse afectada por el número de receptores dentro de la comunidad a que el individuo pertenece (zona geográfica, grupo étnico). Si el cobro de la prestación es generalizado, se reduce significativamente el estigma relacionado con el hecho de haberla solicitado. A su vez, ello contribuye a que la información sobre el programa pertinente se difunda mediante la red de contactos personales, con lo cual disminuyen los costos individuales de obtención y procesamiento de ella. ${ }^{4}$

Dado que no es posible observar directamente la valoración que hacen los hogares o las personas de las prestaciones y de los costos conexos, en los análisis empíricos se utilizan aproximaciones representativas (proxies) de los mismos. A continuación se describen las principales variables utilizadas en los antecedentes empíricos disponibles y los factores determinantes con que ellas guardan relación.

a) Escolaridad del jefe o del hogar. Por una parte, se prevé que a mayor educación, más facilidad para procesar la información relativa al procedimiento de solicitud, con lo cual disminuyen los costos de transacción. No obstante, quienes poseen un mayor nivel de escolaridad tienen mejores oportunidades futuras de aumentar sus ingresos, de manera que el menor plazo de duración de un beneficio determinado afectará negativamente la postulación al programa. Por ello, los efectos de la educación en esta materia son ambiguos. Algunos autores han encontrado una relación negativa entre el nivel de escolaridad y la probabilidad de presentarse (Blank y Ruggles, 1996; Riphahn, 2001), mientras que otros no encontraron una relación significativa (Kayser y Frick, 2001; Terracol, 2002).

b) Propiedad de la vivienda. En línea con la escolaridad, las personas que son propietarias de la vivienda en que residen tienen en promedio mayores ingresos potenciales, por lo que se prevé que requieran asistencia durante períodos más cortos de tiempo,

\footnotetext{
${ }^{4} \mathrm{Al}$ considerar los factores en su conjunto, debe tenerse en cuenta que los costos de transacción y de estigma son mayores al momento de solicitar la prestación, de manera que si los posibles beneficiarios esperan recibirla durante un corto período de tiempo, podrían evaluar que los costos de postular al programa superan los beneficios conexos.
}

resultando menos probable que soliciten prestaciones sociales dados los costos que ello supone.

c) Tipo de hogar. En varios estudios se ha constatado que hay un mayor nivel de postulación a programas sociales entre los hogares monoparentales, los que generalmente son de jefatura femenina. Ello podría obedecer a que tienen menos oportunidades de encontrar un trabajo de ingreso adecuado, ya que como el cuidado de los menores está a cargo de una sola persona adulta se requiere un horario laboral flexible y reducido. En su defecto, ello implica contratar servicios de cuidado, lo que se traducirá en un costo de oportunidad elevado. A su vez, se ha comprobado que las mujeres jefas de hogar suelen solicitar prestaciones sociales con mayor frecuencia que los hombres. De acuerdo con Riphahn (2001), el nivel de estigma social depende de la edad y el sexo del jefe de hogar. Esto es, se percibe como más estigmatizador que un hombre maduro no sea capaz de proveer ingresos suficientes para el hogar que en el caso de una mujer.

d) Presencia de menores en el hogar. En la misma línea anterior, la presencia de niños en el hogar por lo general afecta positivamente las probabilidades de inscribirse en programas sociales, ya que esta puede mitigar los sentimientos de culpabilidad y de estigma (Duclos, 1995).

e) Etnia. En relación a la etnia, se prevé que entre las personas que pertenecen a minorías étnicas aumenta la probabilidad de solicitar prestaciones sociales. Por una parte, es posible que sus expectativas de inserción en el mercado de trabajo y de generación de ingresos sean reducidas debido a las prácticas discriminatorias que enfrentan estos grupos y que se expresan en altas tasas de desempleo específicas y menores remuneraciones relativas. Por otra, los costos en materia de estigma podrían ser inferiores en la medida en que los inscritos representen una proporción elevada del grupo de pares. ${ }^{5}$

f) Edad. En el caso de que un programa social sea accesible para todos los grupos etarios, se prevé que la tasa de participación de los jóvenes será más elevada que la del resto. Es posible que los adultos mayores enfrenten costos más altos si el proceso de postulación es relativamente complejo. Asimismo,

\footnotetext{
${ }^{5}$ Kayser y Frick (2001) sostuvieron que es menos probable que los inmigrantes soliciten prestaciones sociales porque enfrentan un mayor nivel de estigma, así como barreras de idioma y escasa familiaridad con el sistema de protección social. Todos estos factores se agudizan en caso de que el hogar no cuente con un permiso de residencia legal.
} 
en este grupo podrían predominar las actitudes de mayor autonomía y una menor propensión a aceptar prestaciones que no procedan del propio trabajo.

g) Zona geográfica. Otro factor que podría dar cuenta de la falta de respuesta a los programas sociales es el tamaño de la localidad a la que pertenecen los posibles beneficiarios. Es probable que en las comunidades pequeñas aumente la presencia de estigmas, ya que en este caso es más difícil ocultar que se está recibiendo una prestación.

h) Cobro de otros beneficios. Si un hogar determinado ya recibe algún tipo de transferencia, aumentará la probabilidad de inscripción en nuevos programas. La experiencia previa en procesos de postulación similares le permitirá reducir los requerimientos de información y otros costos conexos. A su vez, el costo de estigma para un hogar que ya está recibiendo beneficios sociales es menos significativo (Kayser y Frick, 2001).

i) Porcentaje de beneficiarios en el barrio. Según Mood (2005), la mayoría de los factores determi- nantes mencionados puede atribuirse parcialmente a ciertas normas específicas de los grupos. Si el hecho de recibir prestaciones sociales es algo común, y si los miembros de un grupo se identifican e interactúan más entre sí que con otros individuos, la propensión a solicitarlas debiera ser elevada. Una de las variables representativas más usuales es el porcentaje de hogares que cobran el beneficio en el barrio al que pertenecen. Ello ha sido constatado luego de amplios controles por Bertrand, Luttmer y Mullainathan (2000) en Estados Unidos, Terracol (2002) en Francia y Mood (2004) en Suecia.

En lo que se refiere al método de estimación, para calcular la probabilidad de que los hogares elegibles soliciten una determinada prestación social, generalmente en los estudios se utilizan modelos probit (Riphahn, 2001; Kayser y Frick, 2001; Fuchs, 2007) o logit (Mood, 2005).

En el cuadro 1 se resumen las principales variables empleadas en los antecedentes descritos, los factores determinantes con que guardan relación y el signo de la estimación pertinente.

\section{IV}

\section{Modelo de análisis}

Siguiendo a Blundell, Fry y Walker (1988), se considera un modelo simplificado en que las personas evalúan los beneficios y costos pecuniarios y no pecuniarios a que da lugar la postulación al programa.

$$
U\left[y+B\left(y, z^{*}\right), z\right]-C(y, z)>U(y, z)
$$

donde $y$ representa el ingreso original del hogar, $B=B\left(y, z^{*}\right)$ la prestación que le corresponde a ese hogar en función del ingreso $y$ y de $z^{*}$, que representa el vector de características observables que determinan su otorgamiento.

Por otra parte, $z$ representa el vector de características que determinan la decisión de postular y $C$ ( ) los costos de presentarse, que también es función de y y de $z$.

Si se elige una especificación lineal para las funciones $U$ y $C$, se tiene que

$$
\begin{gathered}
U\left[y+B\left(y, z^{*}\right), z\right]=a_{0}+a_{1}(y+B)+a_{2}^{\prime} z+e_{T}=U_{T} \\
U[y, z]=a_{0}+a_{1} y+a_{2}^{\prime} z+e_{0}=U_{0} \\
-C(y, z)=b_{0}+b_{1} y+b_{2}^{\prime} z+\mu
\end{gathered}
$$

donde $\mathrm{e}_{\mathrm{T}}, \mathrm{e}_{0} \mathrm{y} \mu$ son los factores inobservables específicos de cada hogar.

Haciendo la diferencia, y suponiendo las especificaciones lineales, entre los lados derecho e izquierdo de la ecuación (1) se tiene que

$$
\begin{gathered}
U_{T}-C-U_{0}=b_{0}+a_{1} B+b_{1} y+b_{2}^{\prime} z+v \\
\text { donde } v=e_{T}+\mu-e_{0}
\end{gathered}
$$

La probabilidad de postular es entonces:

$$
P\left(U_{T}-C-U_{0}>0\right)=P\left[v>-\left(b_{0}+a_{1} B+b_{1} y+b_{2}^{\prime} z\right)\right]
$$

Por lo tanto, en el caso del hogar "iésimo" dicha probabilidad puede expresarse como la función de distribución acumulada tal en que

$$
P_{i}=F\left(B_{i}, y_{i}, z_{i}\right)
$$

donde la elección de F (.) depende del supuesto sobre la distribución de $v$ entre los hogares.

Cabe destacar que $C$ es independiente de $B$, lo que implica que existen costos fijos por aplicar (esfuerzo, 


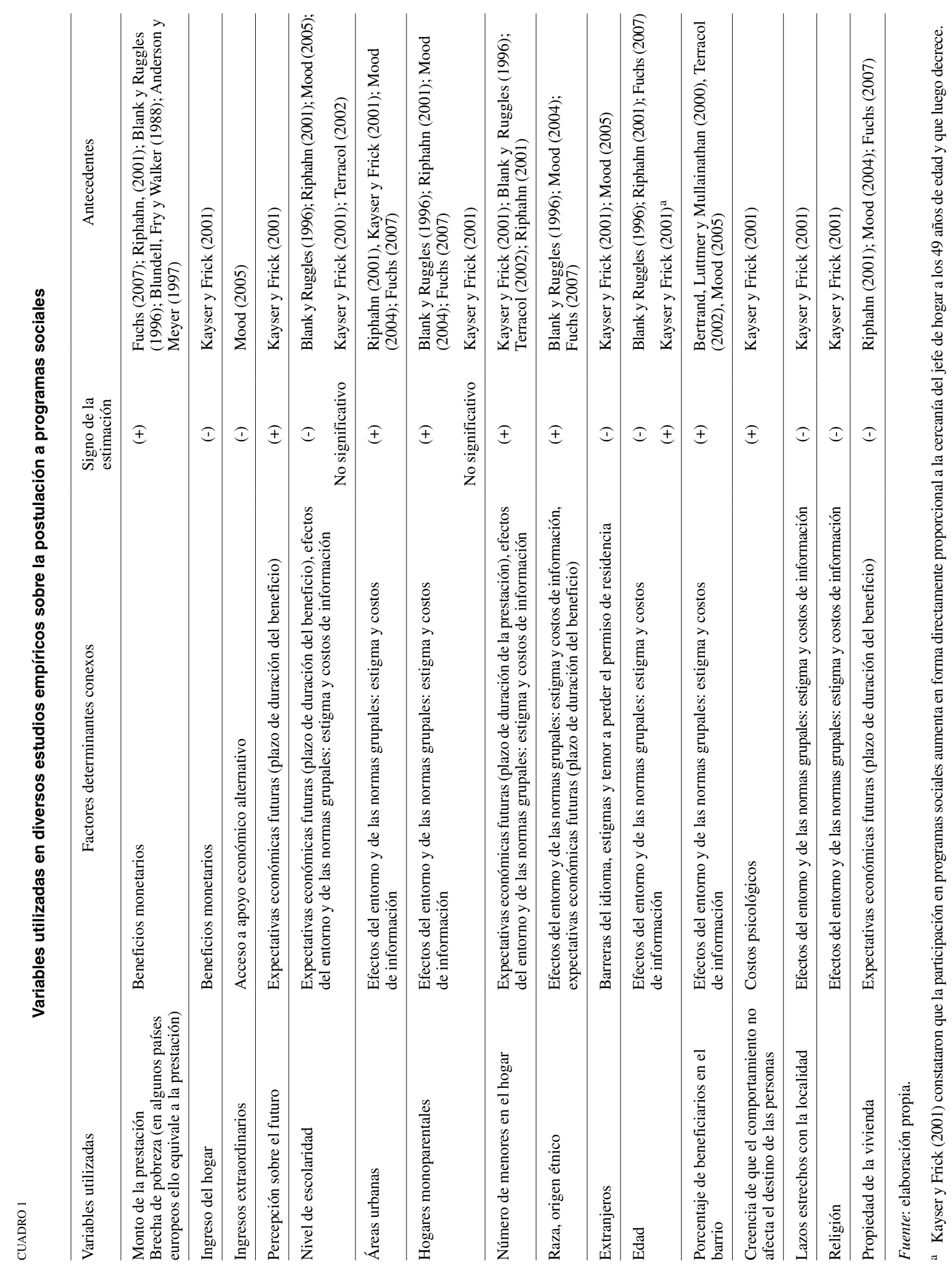


estigma) que varían entre los hogares según las características de $z$ y del ingreso $y$. $C$ (.) depende positivamente de $y$, porque se supone que los hogares de ingreso más elevado se sentirán más estigmatizados a la hora de inscribirse en un programa focalizado. La probabilidad de postulación será decreciente en $y$, dado $B$, tanto por el hecho de que el beneficio relativo previsto es menor como porque los costos de la solicitud en materia de estigma son superiores. Para un nivel dado de $y$, cuanto más elevada sea $B$, mayor será la posibilidad de que los beneficios compensen con creces los costos. Por lo tanto, la probabilidad de postular será creciente en $B$ dados $y$ y $z$.

Las características de los hogares reflejadas en el vector $z$ que fueron consideradas en este estudio son las siguientes (véase el Apéndice):
- Departamento/ barrio

- $\quad$ Nivel de riqueza

- Materiales del techo de la vivienda

- Relación entre el monto de la prestación y el ingreso total del hogar

- Cobro de otras prestaciones del Banco de Previsión Social (BPs) ${ }^{6}$

- Zona geográfica

- Jefe de hogar mayor de 65 años

- Raza

- Hogar monoparental

- Número de menores en el hogar

- Nivel de escolaridad del hogar

- $\quad$ Propiedad de la vivienda

- Jefe de hogar desocupado o inactivo

V

\section{EI Plan de Atención Nacional a la Emergencia Social: aspectos institucionales, criterios de elegibilidad y procedimiento de inscripción}

En este apartado se resumen, siguiendo a Amarante, Burdín y Vigorito (2008), las principales características del PANES, el marco institucional y administrativo y particularmente los requisitos de elegibilidad, así como el procedimiento de inscripción que debían realizar los hogares.

\section{Aspectos generales}

El Plan de Atención Nacional a la Emergencia Social del Uruguay consistió de un conjunto de políticas sociales orientadas a los hogares de muy bajo ingreso. La población objetivo del programa correspondió al primer quintil de personas bajo la línea de pobreza (8\%) y abarcó a la totalidad de las personas en situación de indigencia $(4,2 \%)$. El Plan fue creado mediante la ley 17.869, sancionada en mayo de 2005.

En la documentación oficial sobre el PANES se establece que tuvo dos finalidades básicas: ${ }^{7}$ por una

\footnotetext{
6 Organismo encargado de gestionar el conjunto de prestaciones sociales (contributivas y no contributivas) del Uruguay.

7 Para mayores detalles, véase Ministerio de Desarrollo Social [en línea] www.mides.gub.uy
}

parte, proporcionar asistencia contingente mediante una transferencia monetaria (el Ingreso Ciudadano) y subvenciones para el consumo alimentario; por otra, ofrecer una propuesta de más largo plazo por medio de intervenciones de capacitación, educación y alfabetización, junto con experiencias de participación social y laboral, aunque el desarrollo de estas actividades tuvo un carácter más acotado.

\section{Criterios de elegibilidad}

Para postular al programa, los hogares tuvieron que completar un formulario genérico entregado en el Ministerio de Desarrollo Social (MIDES), que incluía una declaración de ingresos y una enumeración de los miembros del hogar y de sus respectivas cédulas de identidad.

Mediante una subsiguiente visita de funcionarios del MIDES, se reunía información detallada sobre las características del hogar. Además, cuando se inició el programa, en mayo de 2005, se eligieron algunas zonas de mayor carencia en que se realizó un empadronamiento, completando al mismo tiempo los formularios de inscripción y de visita. Este procedimiento abarcó 12.000 hogares y se denominó "desembarcos". Los datos se ingresaron en el MIDEs y fueron transferidos 
al Banco de Previsión Social, donde se cotejó la información contenida en los formularios con la proveniente de los registros de la seguridad social. En el artículo $6^{\circ}$ de la ley 17.869 se establece que: "Las prestaciones se otorgarán a los hogares cuyos ingresos por todo concepto, exceptuándose las asignaciones familiares, prestaciones por invalidez y vejez, al mes de marzo de 2005, no superen los $\$ 1.300$ (mil trescientos pesos uruguayos) por persona y presenten carencias críticas en sus condiciones de vida".
Entre los hogares cuyo ingreso mensual no superaba un límite máximo de 1.300 pesos per cápita, sea declarado o de acuerdo con los registros del BPS, se elegía a los beneficiarios sobre la base de un puntaje determinado mediante la combinación lineal del conjunto de características del hogar que mide el índice de carencias críticas (ICC). Ingresaban al programa aquellos cuyo puntaje superaba un umbral variable según la región. ${ }^{8}$

En el PANES se inscribieron aproximadamente 131.000 hogares, de los cuales se aprobó a cerca de 80.000. ${ }^{9}$

\section{VI}

\section{Fuentes de información y estadísticas descriptivas}

Las fuentes de información utilizadas en el presente estudio fueron la Encuesta Nacional de Hogares Ampliada (ENHA) 2006 y la Encuesta Continua de Hogares 2007. Ambas son representativas del total nacional y contienen información socioeconómica sobre hogares y personas.

Para determinar la elegibilidad de los hogares se utilizaron dos criterios:

i) Hogares ubicados en el primer quintil bajo la línea de pobreza, tal como se definió originalmente la población objetivo. Este criterio solo es aplicable al país urbano. ${ }^{10}$

ii) Hogares cuyo puntaje según el índice de carencias críticas supere el umbral correspondiente y en que el ingreso per cápita, de acuerdo con los criterios establecidos en virtud de la ley pertinente, sea inferior a 1.300 pesos. En este caso, la estimación se realizó para la totalidad del país.

En el cuadro A-1 se presentan las estadísticas descriptivas de las variables utilizadas. Como se observa en el cuadro 2, según el criterio utilizado el porcentaje de hogares elegibles que no postularon al programa osciló entre un $17 \%$ y un $22 \%$, lo que equivale aproximadamente a 9.500 hogares. El mayor porcentaje estimado de acuerdo con el segundo criterio obedeció a que en este caso se incluyó el medio rural, cuya participación en este tipo de programas suele ser menos significativa.

Estos niveles de no solicitud son relativamente bajos en comparación con los antecedentes de los países de la OCDE. ${ }^{11}$ De cualquier modo, las estimaciones de que se dispone sobre otros países generalmente se refieren al porcentaje de hogares o personas elegibles que no cobran las prestaciones y no el de aquellos que no las solicitan, como en el caso del presente estudio. Ello implica que para determinar el nivel de non take-up incluyen los

\footnotetext{
${ }^{8}$ La metodología utilizada para calcular el índice de carencias críticas se describe en Amarante, Arim y Vigorito (2006).

${ }^{9}$ Cabe destacar que el número de hogares aprobados alcanzó aproximadamente el doble de lo planificado inicialmente.

${ }^{10}$ Ello obedece a que la pobreza se calcula únicamente en las zonas urbanas, ya que en el medio rural no se incluye el valor locativo en el ingreso del hogar y por lo tanto los ingresos de ambos no son estrictamente comparables.

${ }^{11}$ Véase Hernanz, Malherbert y Pellizzari (2004) para un análisis sistemático de estas estimaciones.
}

\begin{tabular}{lrr}
\hline & Total \\
& $\begin{array}{c}\text { No } \\
\text { inscritos }\end{array}$ & 16,9 \\
Primer quintil bajo la línea de pobreza & 83,1 & 100,0 \\
Puntaje según índice de carencias críticas e ingreso per cápita inferior a 1.300 pesos & $78,4 \quad 21,6$ & 100,0 \\
\hline
\end{tabular}

Fuente: elaboración propia sobre la base de la Encuesta Nacional de Hogares Ampliada 2006. 
errores cometidos en el proceso administrativo y en la aplicación del instrumento de focalización, es decir, el número de hogares elegibles que solicitaron el beneficio y que fueron rechazados equivocadamente.

Debe tenerse presente, como lo señalan Amarante y otros (2007), que la identificación de los hogares elegibles mediante la Encuesta Nacional de Hogares Ampliada no está exenta de limitaciones metodológicas. En primer lugar, no se dispone de información sobre los hogares previo a la implementación del programa, de manera que es posible que el ingreso declarado en la encuesta difiera del que percibían al momento de postular al PANES. Las mismas consideraciones son válidas para el índice de carencias críticas, ya que las condiciones de vida de los hogares pueden haber variado entre la fecha de solicitud y la de realización del sondeo. Segundo, también es posible que haya diversos grados de subdeclaración del ingreso, tanto en la encuesta como al momento de solicitar el beneficio, puesto que los hogares conocían las condiciones de elegibilidad del Plan. Por último, en el caso de los hogares que recibieron las prestaciones del PANES, se supone que de no ser beneficiarios del Plan no habrían cambiado su comportamiento laboral y que, por lo tanto, su ingreso sería igual al que percibían sin la transferencia del Ingreso Ciudadano. ${ }^{12}$

Pese a estas dificultades, se considera que la información disponible en la ENHA 2006 permite una aproximación razonable a la determinación de las condiciones de elegibilidad para el programa. Asimismo, en la encuesta se distingue entre hogares inscritos y hogares que efectivamente cobran el beneficio, con lo cual pueden

\footnotetext{
12 No existen evidencias concluyentes en relación con los posibles efectos del PANES en el comportamiento laboral. Amarante, Burdín y Vigorito (2008) evaluaron las repercusiones del programa en la oferta laboral (actividad y horas trabajadas), utilizando una metodología de diseño discontinuo realizada sobre la base de una encuesta de los hogares beneficiarios (grupo de tratamiento) y de los que no recibían las prestaciones (grupo de control) en un entorno reducido del punto de corte del algoritmo. Los autores no encontraron pruebas de que postular al PANES afectara el comportamiento laboral. Borraz y González (2008) tampoco encontraron efectos en la actividad laboral, aunque sí cierta reducción de las horas trabajadas. Estos autores no utilizaron un diseño de encuesta específico para la evaluación del programa, aplicando la metodología de emparejamiento (propensity score matching) con la encuesta de hogares.
}

analizarse directamente los factores determinantes de la decisión de postular. Lo anterior constituye una ventaja respecto de otros estudios de este tipo, en que - como se dijo - ella se modela en forma indirecta, distinguiendo solo entre quienes cobran y no cobran el beneficio. De acuerdo con la tipología definida en la sección II, el presente estudio se ocupa de los factores determinantes de la falta de respuesta primaria, pudiendo aislar los problemas del non take-up secundario.

Dada la importancia que representa el hecho de poder realizar una estimación de los factores determinantes de la no postulación al PANES para la totalidad del país, esto es, incluido el medio rural, el criterio de elegibilidad adoptado para estimar la probabilidad de presentarse fue el que considera conjuntamente el índice de carencias críticas y el límite máximo de ingreso.

El cuadro 3 muestra la proporción de hogares elegibles que no se inscribieron en el PANES. Un $22 \%$ de los que reunían los requisitos de elegibilidad no solicitaron las prestaciones pertinentes, porcentaje que se reduce significativamente en el caso de Montevideo.

Entre los hogares cuya tasa de no postulación fue menos significativa se cuentan los que ya recibían una prestación servida por el BPS (asignaciones, pensiones, jubilaciones), aquellos con jefatura de raza negra, ${ }^{13}$ los situados en grandes ciudades y los de mayor presencia de menores. Las zonas rurales presentan tasas de no inscripción extremadamente elevadas, que alcanzan un $46 \%$ de los hogares elegibles. Asimismo, la proporción de no inscritos en el programa fue más elevada en los hogares con jefe de edad avanzada, excepto en Montevideo.

En la categoría de hogares monoparentales, las tasas de no inscripción en el PANEs fueron bajas y en ningún caso superaron el 10\% de los hogares elegibles. En cambio, ellas fueron más significativas en los hogares con vivienda propia y los más educados. Aquellos cuyo jefe se encontraba desocupado o inactivo registraron tasas de no postulación levemente superiores, con excepción de Montevideo.

\footnotetext{
${ }^{13}$ Hogares cuyo jefe se autopercibe como de ascendencia africana.
} 
CUADRO 3

Proporción de hogares no inscritos en el Plan de Atención Nacional a la Emergencia Social del Uruguay, según características, 2006

(En porcentajes de hogares elegibles)

\begin{tabular}{|c|c|c|c|}
\hline & Total país & Montevideo & Interior \\
\hline Total & 21,64 & 12,74 & 24,13 \\
\hline \multicolumn{4}{|c|}{ Recibe otros beneficios del Banco de Previsión Social } \\
\hline No & 28,58 & 18,37 & 32,27 \\
\hline Sí & 19,91 & 10,95 & 22,25 \\
\hline \multicolumn{4}{|l|}{ Jefe de hogar de raza negra } \\
\hline No & 23,16 & 14,13 & 25,49 \\
\hline Sí & 15,51 & 8,48 & 18,11 \\
\hline \multicolumn{4}{|l|}{ Localidades } \\
\hline 5.000 o más habitantes & 12,95 & - & 13,05 \\
\hline Menos de 5.000 habitantes & 33,11 & - & 33,11 \\
\hline Rurales & 46,35 & - & 46,35 \\
\hline \multicolumn{4}{|l|}{ Número de menores } \\
\hline Hogares sin menores & 54,23 & 33,33 & 54,33 \\
\hline Presencia de un menor & 23,73 & 12,07 & 25,27 \\
\hline Presencia de dos menores & 20,53 & 16,14 & 21,67 \\
\hline Presencia de tres menores & 19,24 & 12,69 & 21,22 \\
\hline Presencia de cuatro menores o más & 17,26 & 11,95 & 19,49 \\
\hline \multicolumn{4}{|l|}{ Jefe de hogar mayor de 65 años } \\
\hline No & 19,26 & 12,83 & 21,24 \\
\hline Sí & 47,15 & 6,61 & 48,55 \\
\hline \multicolumn{4}{|l|}{ Hogar monoparental } \\
\hline No & 24,65 & 14,33 & 27,38 \\
\hline Sí & 9,36 & 7,48 & 10,00 \\
\hline \multicolumn{4}{|l|}{ Hogar con vivienda propia } \\
\hline No & 17,58 & 12,52 & 19,49 \\
\hline Sí & 32,11 & 14,88 & 33,52 \\
\hline \multicolumn{4}{|l|}{ Jefe de hogar desocupado/inactivo } \\
\hline No & 21,00 & 14,15 & 23,09 \\
\hline Sí & 22,95 & 9,20 & 26,12 \\
\hline \multicolumn{4}{|l|}{ Escolaridad } \\
\hline Inferior a nueve años & 20,66 & 11,13 & 23,38 \\
\hline Nueve años o más & 28,92 & 26,52 & 29,50 \\
\hline
\end{tabular}

Fuente: elaboración propia sobre la base de la Encuesta Nacional de Hogares Ampliada 2006.

Nota: No incluye las variables riqueza y material de techos, puesto que carecen de una interpretación teórica en particular y en el modelo se utilizan meramente como variables de control. Para mayores detalles, véase el cuadro A-1. 


\section{VII}

\section{Resultados preliminares}

En el cuadro 4 se presentan los resultados del modelo probit basado en la ENHA 2006 mediante el cual se estimó la probabilidad de que un hogar elegible solicitara el Ingreso Ciudadano. ${ }^{14}$ Dado que el beneficio se otorgó a los hogares, la estimación se realizó precisamente a ese nivel.

Siguiendo a Fuchs (2007), se realizaron dos estimaciones: una en que se incluyó la situación de actividad del jefe de hogar y otra en que no se hizo, en virtud de la posible existencia de sesgos de selección. Ambos modelos se estimaron para la totalidad del país, Montevideo y el interior.

En las estimaciones correspondientes a todo el país se incluyeron variables ficticias (dummies) por departamento, a fin de controlar los posibles efectos idiosincrásicos vinculados al lugar de residencia en la probabilidad de postulación. Con el mismo propósito, en el caso de Montevideo se utilizaron variables por barrio. Asimismo, se incluyeron controles sobre el nivel de riqueza, medido según la disponibilidad de bienes durables (riqueza) y los materiales de construcción de la vivienda (techoprecario). ${ }^{15}$

En general, las variables afectan la probabilidad de postulación al PANES con el signo esperado, en forma significativa y son compatibles con los resultados obtenidos en otros estudios.

La relación entre el monto de la prestación y el ingreso total del hogar (beneficio) afecta positivamente la probabilidad de inscripción. En este sentido, los hogares donde el beneficio que ofrecía el programa era importante en términos relativos presentaron mayor número de solicitudes que el resto, en línea con los resultados de otras investigaciones (Anderson y Meyer, 1997; Blundell, Fry y Walker, 1988).

Por otra parte, la probabilidad de postulación fue mayor en los hogares que ya percibían algún tipo de prestación del Banco de Previsión Social (benefbps), apoyando la idea de que la experiencia previa en pro-

\footnotetext{
${ }^{14}$ En el cuadro A-2 se aprecian las estimaciones del modelo correspondientes a 2007. En general, los coeficientes estimados no presentaron una variación significativa respecto de 2006.

${ }^{15}$ A fin de reflejar el número de bienes durables existentes en el hogar, se construyó un índice de riqueza en que se agregaron ambas variables, utilizando ponderadores obtenidos mediante un análisis factorial. Para mayores detalles metodológicos, véase el Apéndice.
}

cedimientos de solicitud similares contribuyó a reducir los costos de procesamiento de la información relativa al PANES.

Los hogares residentes en pequeñas localidades urbanas ${ }^{16}$ (menos5000) y muy particularmente en las zonas rurales (rural) presentaron una menor probabilidad de inscripción que los pertenecientes a grandes ciudades. En las ciudades pequeñas, la imposibilidad de conservar el anonimato puede exacerbar el estigma que por lo general se asocia al hecho de postular a programas focalizados. En las localidades rurales, las dificultades de acceso a la información y los mayores costos de traslado contribuyen a que sea más costoso solicitar las prestaciones del caso. Los efectos encontrados fueron similares a los verificados en las investigaciones revisadas.

Por otra parte, se observó un efecto negativo de la edad del jefe de hogar en la probabilidad de postulación, sobre todo cuando este superaba los 65 años de edad (may65). Esto podría indicar que en la población de edad más avanzada aumentan los costos sociales y psicológicos, así como las dificultades para procesar la información necesaria. Cabe destacar que el resultado se invierte en Montevideo, donde el coeficiente fue positivo. Ello obedeció tal vez a la mitigación del efecto estigma en este grupo etario cuando las interacciones sociales son más impersonales, como efectivamente sucede en la capital del país.

Los hogares con jefe de raza negra (afro) presentaron una mayor probabilidad de inscribirse en el PANES. Este efecto se observó en la totalidad del país y en Montevideo, perdiendo importancia en la estimación correspondiente al interior. ${ }^{17}$ En este sentido, si la proporción de solicitantes de este grupo étnico es relativamente elevada, la información se habría propagado mediante la red de contactos personales, reduciendo así el efecto estigma e incentivando la postulación al programa.

La condición de hogar monoparental (monoparental) y la presencia de menores de 18 años (men18) afectan positivamente la probabilidad de solicitar el beneficio. En el primer caso, la existencia de un solo receptor potencial de ingresos contribuiría a deteriorar

\footnotetext{
${ }^{16}$ Centros urbanos de menos de 5.000 habitantes.

${ }^{17}$ Esto podría obedecer a que en Montevideo hay mayor presencia de personas de ascendencia africana.
} 
CUADRO 4

Modelo probit de la probabilidad de postular al Plan de Atención Nacional a la Emergencia Social del Uruguay, 2006

(Efectos marginales)

\begin{tabular}{|c|c|c|c|c|c|c|}
\hline \multirow[t]{2}{*}{ Variables explicativas } & \multicolumn{2}{|c|}{ Total país } & \multicolumn{2}{|c|}{ Montevideo } & \multicolumn{2}{|c|}{ Interior } \\
\hline & Modelo 1 & Modelo 2 & Modelo 1 & Modelo 2 & Modelo 1 & Modelo 2 \\
\hline Beneficio & $\begin{array}{l}0,119 \\
(0,007)^{* * * *}\end{array}$ & $\begin{array}{l}0,12 \\
(0,007)^{* * * *}\end{array}$ & $\begin{array}{l}0,126 \\
(0,017)^{* * * *}\end{array}$ & $\begin{array}{l}0,13 \\
(0,017)^{* * * *}\end{array}$ & $\begin{array}{l}0,124 \\
(0,008)^{* * * *}\end{array}$ & $\begin{array}{l}0,124 \\
(0,008)^{* * * *}\end{array}$ \\
\hline Benefbps & $\begin{array}{l}0,111 \\
(0,006)^{* * *}\end{array}$ & $\begin{array}{l}0,112 \\
(0,006)^{* * *}\end{array}$ & $\begin{array}{l}0,115 \\
(0,010)^{* * *}\end{array}$ & $\begin{array}{l}0,117 \\
(0,011)^{* * *}\end{array}$ & $\begin{array}{l}0,1 \\
(0,007)^{* * *}\end{array}$ & $\begin{array}{l}0,101 \\
(0,007)^{* * *}\end{array}$ \\
\hline Menos5000 & $\begin{array}{l}-0,094 \\
(0,006)^{* * *}\end{array}$ & $\begin{array}{l}-0,093 \\
(0,006)^{* * *} *\end{array}$ & & & $\begin{array}{l}-0,09 \\
(0,006) * * *\end{array}$ & $\begin{array}{l}-0,09 \\
(0,006)^{* * *}\end{array}$ \\
\hline Rural & $\begin{array}{l}-0,264 \\
(0,008) * * *\end{array}$ & $\begin{array}{l}-0,264 \\
(0,008) * * *\end{array}$ & & & $\begin{array}{l}-0,262 \\
(0,008)^{* * *}\end{array}$ & $\begin{array}{l}-0,262 \\
(0,008)^{* * *}\end{array}$ \\
\hline Men 18 & $\begin{array}{l}0,04 \\
(0,001)^{* * *}\end{array}$ & $\begin{array}{l}0,04 \\
(0,001)^{* * * *}\end{array}$ & $\begin{array}{l}0,023 \\
(0,002)^{* * *}\end{array}$ & $\begin{array}{l}0,022 \\
(0,002)^{* * *}\end{array}$ & $\begin{array}{l}0,048 \\
(0,002)^{* * *}\end{array}$ & $\begin{array}{l}0,048 \\
(0,002)^{* * * *}\end{array}$ \\
\hline May65 & $\begin{array}{l}-0,126 \\
(0,010)^{* * *}\end{array}$ & $\begin{array}{l}-0,119 \\
(0,009)^{* * *}\end{array}$ & $\begin{array}{l}0,046 \\
(0,020)^{* *}\end{array}$ & $\begin{array}{l}0,054 \\
(0,018)^{* * *}\end{array}$ & $\begin{array}{l}-0,119 \\
(0,010)^{* * *}\end{array}$ & $\begin{array}{l}-0,117 \\
(0,010)^{* * *}\end{array}$ \\
\hline Climaeduc & $\begin{array}{l}-0,004 \\
(0,001)^{* * *}\end{array}$ & $\begin{array}{l}-0,004 \\
(0,001)^{* * *}\end{array}$ & $\begin{array}{l}-0,008 \\
(0,002)^{* * *}\end{array}$ & $\begin{array}{l}-0,008 \\
(0,002)^{* * *}\end{array}$ & $\begin{array}{l}-0,002 \\
(0,001)^{*}\end{array}$ & $\begin{array}{l}-0,002 \\
(0,001)^{* *}\end{array}$ \\
\hline Afro & $\begin{array}{l}0,014 \\
(0,005)^{* * * *}\end{array}$ & $\begin{array}{l}0,014 \\
(0,005)^{* * * *}\end{array}$ & $\begin{array}{l}0,047 \\
(0,007)^{* * * *}\end{array}$ & $\begin{array}{l}0,048 \\
(0,007)^{* * * *}\end{array}$ & $\begin{array}{l}-0,004 \\
-0,006\end{array}$ & $\begin{array}{l}-0,004 \\
-0,006\end{array}$ \\
\hline Monoparental & $\begin{array}{l}0,086 \\
(0,005)^{* * * *}\end{array}$ & $\begin{array}{l}0,088 \\
(0,004)^{* * * *}\end{array}$ & $\begin{array}{l}0,031 \\
(0,008)^{* * * *}\end{array}$ & $\begin{array}{l}0,037 \\
(0,008)^{* * * *}\end{array}$ & $\begin{array}{l}0,106 \\
(0,005)^{* * * *}\end{array}$ & $\begin{array}{l}0,107 \\
(0,005)^{* * *}\end{array}$ \\
\hline Desoc/inact & $\begin{array}{l}0,012 \\
(0,005)^{* *}\end{array}$ & & $\begin{array}{l}0,03 \\
(0,007)^{* * * *}\end{array}$ & & $\begin{array}{r}0,004 \\
-0,006\end{array}$ & \\
\hline Propietario & $\begin{array}{l}-0,058 \\
(0,005)^{* * *}\end{array}$ & $\begin{array}{l}-0,058 \\
(0,005)^{* * *}\end{array}$ & $\begin{array}{l}-0,016 \\
-0,011\end{array}$ & $\begin{array}{l}-0,012 \\
-0,011\end{array}$ & $\begin{array}{l}-0,063 \\
(0,005)^{* * *}\end{array}$ & $\begin{array}{l}-0,063 \\
(0,005)^{* * * *}\end{array}$ \\
\hline Riqueza & $\begin{array}{l}-0,103 \\
(0,003) * * *\end{array}$ & $\begin{array}{l}-0,103 \\
(0,003) * * *\end{array}$ & $\begin{array}{l}-0,077 \\
(0,006)^{* * *}\end{array}$ & $\begin{array}{l}-0,077 \\
(0,006)^{* * *}\end{array}$ & $\begin{array}{l}-0,115 \\
(0,003) * * *\end{array}$ & $\begin{array}{l}-0,115 \\
(0,003)^{* * *}\end{array}$ \\
\hline Techoprecario & $\begin{array}{l}0,066 \\
(0,004)^{* * *}\end{array}$ & $\begin{array}{l}0,066 \\
(0,004)^{* * * *}\end{array}$ & $\begin{array}{l}0,048 \\
(0,007)^{* * * *}\end{array}$ & $\begin{array}{l}0,046 \\
(0,007)^{* * *}\end{array}$ & $\begin{array}{c}0,073 \\
(0,005)^{* * * *}\end{array}$ & $\begin{array}{l}0,073 \\
(0,005)^{* * *}\end{array}$ \\
\hline $\mathrm{N}^{\mathrm{o}}$ de observaciones & 41974 & 41974 & 8293 & 8293 & 32754 & 32754 \\
\hline Pseudo R2 & 0,213 & 0,2129 & 0,1562 & 0,154 & 0,2294 & 0,2294 \\
\hline
\end{tabular}

Fuente: elaboración propia sobre la base de la Encuesta Nacional de Hogares Ampliada 2006.

Nota: Estándares de error sólidos entre paréntesis; * significativo al 10\%, ** significativo al $5 \%$, *** significativo al $1 \%$.

Variables explicativas: Beneficio = relación entre monto de la prestación e ingreso total del hogar; Benefbps $=$ otras prestaciones del Banco de Previsión Social; Menos 5000 = pequeñas localidades urbanas; Rural = zonas rurales; Men18 = número de menores de 18 años; May65 = jefe de hogar mayor de 65 años; Climaeduc = escolaridad del hogar; Afro = hogar con jefatura de raza negra; Monoparental = hogar monoparental;

Desoc/inact $=$ jefe de hogar desocupado o inactivo; Propietario $=$ hogar con vivienda propia; Riqueza $=$ disponibilidad de bienes durables; Techoprecario = materiales de construcción de la vivienda. 
las expectativas de inserción laboral y estimularía la postulación al programa. En el segundo, el mayor número de menores en el hogar aumentaría las posibilidades de éxito en el proceso de aplicación. A su vez, los mecanismos estigmatizadores no afectarían mayormente a este tipo de hogares en comparación con aquellos en que el beneficio se otorga a personas adultas capaces de generar ingresos por sí mismas. Los resultados obtenidos en relación con distintos programas y países por Blank y Ruggles (1996), Riphahn (2001), y Kayser y Frick (2001) fueron similares. ${ }^{18}$

En cambio, la probabilidad de solicitar la prestación está inversamente relacionada con el nivel de escolaridad del hogar (climaeduc), aunque la magnitud del efecto no es significativa. Un resultado similar fue encontrado por Riphahn (2001) en el caso de Alemania. Es posible que el signo obedezca al hecho de que los hogares de mayor capital educacional tendrían mejores expectativas de mejorar su situación económica en el futuro. En forma similar podría interpretarse el efecto negativo en la probabilidad de postular a que da lugar la condición de propiedad de la vivienda (propietario).

Por otra parte, la desocupación o inactividad del jefe de hogar (desoc/inact) aumenta la probabilidad de inscripción. En línea con lo constatado por Fuchs (2007), el hecho de que los coeficientes no varíen de manera significativa al excluir o incluir la variable que identifica si el jefe de hogar se encuentra desocupado o inactivo indicaría que las variables seleccionadas no son altamente endógenas a dicha característica.

\section{VIII}

\section{Comentarios finales}

En el presente documento se abordó el problema de no postulación al PANES de los hogares que satisfacían los requisitos de elegibilidad del programa.

Sobre la base de la Encuesta Nacional de Hogares Ampliada 2006, se estimó que un 22\% de los hogares potencialmente elegibles no había solicitado ingresar al programa. Si bien este guarismo podría llamar la atención, no resulta particularmente elevado en comparación con los antecedentes de los países de la OCDE. De cualquier modo, la proporción fue bastante mayor en las localidades urbanas de menos de 5.000 habitantes (33\%) y sobre todo en el medio rural (46\%).

De acuerdo con las estimaciones del modelo probit, la probabilidad de postulación se vio afectada positivamente por el peso relativo de la transferencia en relación con el ingreso del hogar. El número de menores de 18 años, la recepción de otros beneficios servidos por el BPS, la existencia de un jefe de hogar de raza negra y la condición de hogar monoparental también afectaron en forma positiva la presentación al PANES. En otro orden, la residencia en zonas rurales influyó negativa y significativamente en la probabilidad de inscripción, al igual que la presencia de un jefe de hogar mayor de 65 años. De esta forma, las evidencias respaldarían en gran medida las causas determinantes discutidas en la literatura teó-

\footnotetext{
${ }^{18}$ En este último caso, los autores no encontraron efectos significativos de la situación de hogar monoparental.
}

rica (factores monetarios, costos de información, costos sociales y psicológicos). Asimismo, los resultados son compatibles con los encontrados en estudios similares realizados en los países de la OCDE.

Dado que se trató de una aproximación preliminar, quedan planteadas algunas limitaciones y futuras líneas de profundización del presente artículo. En primer lugar, debido a la falta de información no fue posible analizar el efecto de los "desembarcos" en la probabilidad de postular. En la medida en que se disponga de esa información sería relevante examinarlos, ya que fue un elemento de diseño orientado claramente a reducir las tasas de no inscripción.

Segundo, sería necesario analizar la solidez de los resultados obtenidos mediante otros métodos de estimación que consideren de manera más sistemática la presencia de posibles sesgos de selección. Esto es especialmente importante cuando se analiza el efecto de la situación de actividad económica del jefe de hogar. Es posible que algunas personas alteren su comportamiento laboral a los efectos de cumplir los requisitos de elegibilidad que, en materia de ingreso, exigen los programas sociales focalizados. Esto podría provocar un sesgo en las estimaciones y conducir a una sobrestimación de los efectos de la desocupación como factor determinante de la participación en programas sociales. No obstante, vale decir que en otros estudios en que se ha introducido este tipo de correcciones no se han encontrado diferencias significativas (Fuchs, 2007). 
En tercer lugar, sería particularmente relevante incorporar en forma sistemática al modelo los efectos de las interacciones sociales en la decisión de postular al PANES. El signo encontrado en muchas de las variables utilizadas podría estar reflejando algunas consecuencias de este tipo, como por ejemplo, el mayor número de solicitudes presentadas por jefes de hogar de raza negra. No obstante, esta aproximación preliminar no permitió distinguir con exactitud de qué manera las interacciones sociales afectarían la probabilidad de inscripción de los hogares. En particular, fue imposible medir el peso específico del efecto información y del efecto estigma. La distinción no es trivial desde el punto de vista de las implicaciones de política (Cohen-Cole y Zanella, 2008). Al considerar las interacciones sociales en un modelo de decisión como el utilizado en este trabajo, surgen complejidades que deben abordarse en futuras etapas de la presente investigación (Manski, 1993; Brock y Durlauf, 2001).

Por último, sería deseable avanzar en la interiorización de las decisiones de postulación en las evaluaciones ex ante de los programas sociales del Uruguay. En general, este tipo de estudios suponen la focalización perfecta de las políticas, lo que puede conducir a una apreciación inexacta de sus efectos distributivos y fiscales. Esto parece ser particularmente importante, por ejemplo, en el contexto de aplicación del nuevo régimen uruguayo de asignaciones familiares.
De cualquier forma, el análisis precedente deja planteadas interesantes implicaciones de política social. Como se dijo, la preocupación por la adecuada focalización de los programas sociales se ha centrado excesivamente en excluir de los beneficios a las personas que superan los límites máximos de ingreso y riqueza preestablecidos. Sin embargo, se ha prestado menos atención al hecho de que muchos programas no alcanzan a la totalidad de su población objetivo, en parte debido a que un segmento de los posibles beneficiarios ni siquiera llega a solicitar las prestaciones. Esto podría ser una desventaja en comparación con esquemas más universales, que debería balancearse respecto de los mayores costos fiscales vinculados a estos últimos.

Por otra parte, como lo señala Van Oorschot (1991), resulta crucial analizar la estructura, diseño y administración de los programas sociales, incluidas la difusión de los mismos y la comunicación de los requisitos y procedimientos para acceder a sus beneficios. La mejora de los sistemas de información, la simplificación de los trámites de postulación y la elección cuidadosa de los mecanismos que determinan la "salida" de los programas son ejemplos de medidas concretas que podrían aplicarse al respecto (Fuchs, 2007). En este sentido, las características de los hogares elegibles que no solicitan las prestaciones ofrecen orientaciones para el estudio de estas medidas.

APÉNDICE

Definición de las variables utilizadas en el modelo ${ }^{19}$

\begin{tabular}{ll}
\hline Beneficio & Relación entre el monto de la prestación y el ingreso total del hogar. \\
Benefbps & Variable binaria que indica si el hogar recibe otras prestaciones del Banco de Previsión Social. \\
Menos5000 & Variable binaria que indica si el hogar reside en localidades urbanas de 5.000 habitantes o más (omitida). \\
Inturbano & Variable binaria que indica si el hogar reside en zonas urbanas de menos de 5.000 habitantes. \\
Rural & Variable binaria que indica si el hogar pertenece a una zona rural. \\
May65 & Variable binaria que indica si el jefe del hogar es mayor de 65 años. \\
Afro & Variable binaria que indica si el jefe de hogar se considera de ascendencia africana. \\
Monoparental & Variable binaria que indica si el hogar se compone de un solo jefe/a más hijos. \\
Men18 & Número de menores de 18 años en el hogar. \\
Climaeduc & Promedio de años de educación formal aprobados por los adultos del hogar. \\
Propietario & Variable binaria que indica si el hogar habita en una vivienda propia. \\
Desoc/inact & Variable binaria que indica si el jefe de hogar se encuentra desocupado o inactivo. \\
Riqueza & Variable construida mediante un análisis factorial que ofrece una aproximación sobre el nivel de riqueza del hogar. \\
Techoprecario & Variable binaria que indica si el material predominante del techo es liviano, sin cielorraso, de quincha o de materiales de \\
& desecho.
\end{tabular}

${ }^{19}$ Asimismo, se utilizaron variables binarias de los 19 departamentos para las estimaciones correspondientes a todo el país y de los barrios en el caso de Montevideo.

\footnotetext{
${ }^{20}$ Cuando en el hogar no hay individuos mayores de 18 años, se consideran los años de escolaridad del jefe de hogar.
} 


\section{Metodología del índice combinado de riqueza}

A fin de obtener una variable representativa del nivel de riqueza de los hogares de todo el país, se construyó un índice de riqueza basado en la disponibilidad de ciertos bienes durables. Los coeficientes de dicho índice se obtuvieron mediante el método de componentes principales.

\section{Ponderadores}

\begin{tabular}{ll}
\hline Calefón & 0,6665 \\
Conexión a televisión por cable & 0,5392 \\
Teléfono fijo & 0,6367 \\
Reproductor de vídeos o DVD & 0,6504 \\
Lavarropas & 0,7232 \\
Microondas & 0,7329 \\
Microcomputador & 0,6489 \\
Automóvil & 0,5421
\end{tabular}

Fuente: elaboración propia sobre la base de la Encuesta Nacional de Hogares Ampliada 2006.
CUADRO A-1

\section{Estadísticas descriptivas, 2006}

\begin{tabular}{lccccc}
\hline & \multicolumn{2}{c}{ Población total } & & \multicolumn{2}{c}{ Población elegible } \\
\cline { 2 - 3 } & Media & Desvío & & Media & Desvío \\
\hline Beneficio & 0,180 & 0,246 & & 0,458 & 0,496 \\
Benefbps & 0,602 & 0,490 & & 0,800 & 0,400 \\
Afro & 0,075 & 0,264 & & 0,198 & 0,399 \\
Montevideo & 0,869 & 0,337 & & 0,656 & 0,475 \\
Inturbano & 0,065 & 0,247 & & 0,211 & 0,408 \\
Rural & 0,066 & 0,247 & & 0,133 & 0,340 \\
Men18 & 0,838 & 1,228 & & 2,932 & 1,911 \\
May65 & 0,276 & 0,447 & & 0,085 & 0,279 \\
Climaeduc & 8,710 & 3,782 & & 6,029 & 2,060 \\
Monoparental & 0,116 & 0,321 & & 0,197 & 0,398 \\
Desoc/inact & 0,354 & 0,478 & & 0,331 & 0,470 \\
Propietario & 0,514 & 0,500 & & 0,279 & 0,449 \\
Riqueza & 2,432 & 1,574 & & 0,476 & 0,712 \\
Techoprecario & 0,111 & 0,314 & & 0,478 & 0,500
\end{tabular}

Fuente: elaboración propia sobre la base de la Encuesta Nacional de Hogares Ampliada 2006.

CUADRO A-2

Modelo probit de la probabilidad de postular al Plan de Atención Nacional a la Emergencia Social, 2007

(Efectos marginales)

\begin{tabular}{|c|c|c|c|c|c|c|}
\hline \multirow{2}{*}{ Variables explicativas } & \multicolumn{2}{|c|}{ Total país } & \multicolumn{2}{|c|}{ Montevideo } & \multicolumn{2}{|c|}{ Interior } \\
\hline & Modelo 1 & Modelo 2 & Modelo 3 & Modelo 4 & Modelo 5 & Modelo 6 \\
\hline \multirow{2}{*}{ Beneficio } & 0,019 & 0,021 & 0,165 & 0,168 & 0,013 & 0,015 \\
\hline & $(0,005)^{* * * *}$ & $(0,006)^{* * *}$ & $(0,017)^{* * *}$ & $(0,017)^{* * *}$ & $(0,004)^{* * * *}$ & $(0,005)^{* * *}$ \\
\hline \multirow[t]{2}{*}{ Benefbps } & 0,1 & 0,102 & 0,13 & 0,128 & 0,085 & 0,09 \\
\hline & $(0,006)^{* * *}$ & $(0,006)^{* * * *}$ & $(0,012)^{* * * *}$ & $(0,012)^{* * * *}$ & $(0,007)^{* * * *}$ & $(0,008)^{* * * *}$ \\
\hline \multirow{2}{*}{ Inturbano } & $-0,038$ & $-0,042$ & & & & \\
\hline & $(0,011)^{* * * *}$ & $(0,011)^{* * * *}$ & & & & \\
\hline \multirow{2}{*}{ Rural } & $-0,177$ & $-0,186$ & & & $-0,106$ & $-0,106$ \\
\hline & $(0,026)^{* * * *}$ & $(0,027)^{* * *}$ & & & $(0,009)^{* * *}$ & $(0,009)^{* * *}$ \\
\hline \multirow{2}{*}{ Men18 } & 0,023 & 0,024 & 0,027 & 0,027 & 0,022 & 0,024 \\
\hline & $(0,001)^{* * * *}$ & $(0,001)^{* * *}$ & $(0,002)^{* * *}$ & $(0,002)^{* * *}$ & $(0,001)^{* * *}$ & $(0,001)^{* * *}$ \\
\hline \multirow{2}{*}{ May65 } & $-0,152$ & $-0,121$ & $-0,082$ & $-0,065$ & $-0,176$ & $-0,142$ \\
\hline & $(0,015)^{* * *}$ & $(0,014) * * *$ & $(0,029) * * *$ & $(0,027) * *$ & $(0,019) * * *$ & $(0,017)^{* * *}$ \\
\hline \multirow{2}{*}{ Climaeduc } & $-0,01$ & $-0,011$ & $-0,001$ & $-0,001$ & $-0,011$ & $-0,012$ \\
\hline & $(0,001)^{* * *}$ & $(0,001)^{* * *}$ & $-0,002$ & $-0,002$ & $(0,001)^{* * *}$ & $(0,001)^{* * *}$ \\
\hline \multirow{2}{*}{ Afro } & 0,013 & 0,013 & 0,013 & 0,013 & 0,022 & 0,023 \\
\hline & $(0,004)^{* * *}$ & $(0,004)^{* * *}$ & $-0,008$ & $(0,008)^{*}$ & $(0,004)^{* * *}$ & $(0,004) * * *$ \\
\hline \multirow{2}{*}{ Monoparental } & 0,042 & 0,049 & 0,098 & 0,1 & 0,025 & 0,033 \\
\hline & $(0,003)^{* * *}$ & $(0,003)^{* * *}$ & $(0,007)^{* * *}$ & $(0,006)^{* * *}$ & $(0,004)^{* * *}$ & $(0,004) * * *$ \\
\hline \multirow[t]{2}{*}{ Desoc/inact } & 0,038 & & 0,018 & & 0,042 & \\
\hline & $(0,003) * * *$ & & $(0,008)^{* *}$ & & $(0,004)^{* * *}$ & \\
\hline \multirow[t]{2}{*}{ Propietario } & $-0,01$ & $-0,007$ & 0,028 & 0,032 & $-0,017$ & $-0,013$ \\
\hline & $(0,003)^{* * *}$ & $(0,003)^{*}$ & $(0,008)^{* * *}$ & $(0,008)^{* * *}$ & $(0,004)^{* * *}$ & $(0,004) * * *$ \\
\hline \multirow[t]{2}{*}{ Riqueza } & $-0,037$ & $-0,039$ & $-0,004$ & $-0,003$ & $-0,04$ & $-0,044$ \\
\hline & $(0,002)^{* * *}$ & $(0,002)^{* * *}$ & $-0,005$ & $-0,005$ & $(0,003) * * *$ & $(0,003) * * *$ \\
\hline \multirow{2}{*}{ Techoprecario } & 0,012 & 0,011 & 0,055 & 0,054 & 0 & $-0,001$ \\
\hline & $(0,003)^{* * *}$ & $(0,003)^{* * *}$ & $(0,007)^{* * *}$ & $(0,007)^{* * *}$ & $-0,004$ & $-0,004$ \\
\hline
\end{tabular}

Fuente: elaboración propia sobre la base de la Encuesta Continua de Hogares 2007.

Nota: Estándares de error sólidos entre paréntesis; $*$ significativo al $10 \%, * *$ significativo al $5 \%$, *** significativo al $1 \%$

Variables explicativas: Beneficio $=$ relación entre monto de la prestación e ingreso total del hogar; Benefbps $=$ otras prestaciones del Banco de Previsión Social; Inturbano = zonas urbanas; Rural = zonas rurales; Men18 = número de menores de 18 años; May65 = jefe de hogar mayor de 65 años; Climaeduc $=$ escolaridad del hogar; Afro = hogar con jefatura de raza negra; Monoparental = hogar monoparental; Desoc/inact $=$ jefe de hogar desocupado o inactivo; Propietario = hogar con vivienda propia; Riqueza $=$ disponibilidad de bienes durables; Techoprecario $=$ materiales de construcción de la vivienda. 
Amarante, V., R. Arim y A. Vigorito (2006), Convenio UDELAR-PANES. Informe Final, Montevideo, Instituto de Economía, Facultad de Ciencias Económicas y de Administración, Universidad de la República.

Amarante, V., G. Burdín y A. Vigorito (2008), "Evaluación cuantitativa del impacto del PANES", documento presentado en las Jornadas de Economía del Banco Central del Uruguay.

Amarante, V. y otros (2007), Una caracterización socioeconómica de la población incluida en el PANES, documento elaborado en el marco del Convenio UdELAR-MIDES, Montevideo.

Anderson, P.M. y D.B. Meyer (1997), "Unemployment insurance take-up rates and the after-tax value of benefits", The Quarterly Journal of Economics, vol. 112, Cambridge, Massachusetts, The MIT Press.

Ashenfelter, O. (1983), "Determining participation in incometested social programs", Journal of the American Statistical Association, vol. 78, No 383, Alexandria, American Statistical Association.

Atkinson, A.B. (1995), "On targeting social security: theory and Western experience with family benefits", Public Spending and the Poor: Theory and Evidence, D. Van de Walle y K. Nead (comps.), Maryland, Banco Mundial/Johns Hopkins University Press.

(1989), "The take-up of social security benefits", Poverty and Social Security, Londres, Harvester Wheatsheaf.

Bertrand, M., E. Luttmer y S. Mullainathan (2000), "Network effects and welfare cultures", Quarterly Journal of Economics, vol. 115, $\mathrm{N}^{\mathrm{o}}$ 3, Cambridge, Massachusetts, The MIT Press.

Blank, R.M. y P. Ruggles (1996), "When do women use aid to families with dependent children and food stamps? The dynamics of elegibility versus participation", The Journal of Human Resources, vol. 31, $\mathrm{N}^{\mathrm{o}}$ 1, Madison, Wisconsin, University of Wisconsin Press.

Blundell, R., B. Fry e I. Walker (1988), "Modelling the take-up of means-tested benefits: the case of housing benefits in the United Kingdom", The Economic Journal, vol. 98, N 390, Oxford, Reino Unido, Blackwell Publishing.

Bollinger, C.R. y M.H. David (2001), "Estimation with response error and nonresponse: food stamp participation in the SIPP", Journal of Business and Economic Statistics, vol. 19, N ${ }^{\circ}$ 2, Alexandria, American Statistical Association.

Borraz, F. y N. González (2008), "PANEs: focalización e impacto", documento presentado en las Jornadas de Economía del Banco Central del Uruguay.

Brock, W. y S. Durlauf (2001), "Interactions-based models", Handbook of Econometrics, J. Heckman y E. Leamer (comps.), vol. 5, Amsterdam, Elsevier.

CEPAL (Comisión Económica para América Latina y el Caribe) (2006), La protección social de cara al futuro: acceso, financiamiento y solidaridad (LC/G.2294(SES.31/3)), Santiago de Chile.

Coady, D., M. Grosh y J. Hoddinott (2004), La focalización de las transferencias en los países en desarrollo: revisión de lecciones y experiencias, Washington, D.C., Banco Mundial.

Cohen-Cole, E. y G. Zanella (2008), "Welfare stigma or information sharing? Decomposing social interaction effects in social benefit use", Quaderni del dipartimento di Economia Politica, № 531, Siena, Universidad de Siena.

Corden, A. (1995), Changing Perspectives on Benefit Take-Up, Heslington, York, Social Policy Research Unit, Universidad de York.

Cornia A. y F. Stewart (1995), "Two errors of targeting", Public spending and the poor: Theory and Evidence, D. Van de Walle y K. Nead (comps.), Maryland, Banco Mundial/Johns Hopkins University Press.

Craig, P. (1991), "Costs and benefits: a review of research on take-up of income-related benefits", Journal of Social Policy, vol. 20, Cambridge, Cambridge University Press.

Currie, J. (2004), "The take-up of social benefits", Discussion Paper, $\mathrm{N}^{\mathrm{o}} 1103$, Bonn, Institute for the Study of Labor (IZA)

Duclos, J.-Y. (1995), "Modelling the take-up of state support", Journal of Public Economics, vol. 58, No 3 , Amsterdam, Elsevier.

Durlauf, S. (2002), "Groups, social influences and inequality: a memberships theory perspective on poverty traps", Working Paper, $\mathrm{N}^{\mathrm{o}}$ 18, Wisconsin, Departamento de Economía, Universidad de Wisconsin.

Durlauf, S. y E. Cohen-Cole (2004), "Social interaction models", Working Paper, $\mathrm{N}^{\circ}$ 8, Wisconsin, Universidad de Wisconsin, febrero.

Elster, J. (1989), El cemento de la sociedad: las paradojas del orden social, Barcelona, Gedisa.

Fuchs, M. (2007), "Social assistance - no, thanks? Empirical analysis of non-take-up in Austria 2003", EUROMOD Working Paper, $\mathrm{N}^{\circ}$ EM4/7, Essex, Institute for Social and Economic Research.

Hernanz, V., F. Malherbert y M. Pellizzari (2004), "Take-up of welfare benefits in OECD countries: a review of the evidence", $O E C D$ Social, Employment and Migration Working Papers, $\mathrm{N}^{\mathrm{o}} 17$, París, Organización de Cooperación y Desarrollo Económicos (OCDE).

Kayser, H.A. y J.R. Frick (2001), "Take it or leave it: (non-) takeup behavior of social assistance in Germany", Schmoller's Jahrbuch - Journal of Applied Social Science Studies, vol. 121, $\mathrm{N}^{\mathrm{o}}$ 1, Berlin.

Lindbeck, A., S. Nyberg y J. Weibull (1999), "Social norms and economic incentives in the welfare state", Quarterly Journal of Economics, vol. 114, N ${ }^{\circ}$ 1, Cambridge, Massachusetts, The MIT Press.

Manski, C. (1993), "Identifications of endogenous social effects: the reflection problem", Review of Economic Studies, vol. 60, № 3 , Londres, London School of Economics and Political Science.

Moffitt, R. (1983), "An economic model of welfare stigma”, American Economic Review, vol. 73, № 5, Nashville, Tennessee, American Economic Association.

Mood, C. (2005), "Take-up Down Under: Hits and Misses of Meantested Benefits in Australia", documento presentado en la HILDA Survey Research Conference (29 y 30 de septiembre), Melbourne, Universidad de Melbourne.

(2004), "Social influence effects on social assistance recipiency", Acta Sociologica, vol. 47, No 2, Thousand Oaks, Sage Publications.

Riphahn, R. (2001), "Rational poverty or poor rationality? The take-up of social assistance benefits", Review of Income and Wealth, vol. 47, Oxford, Reino Unido, Blackwell Publishing.

Sen, A. (1995), "The political economy of targeting", Public Spending and the Poor: Theory and Evidence, D. Van de Walle y $\mathrm{K}$ Nead (comps.), Maryland, Banco Mundial/Johns Hopkins University Press.

Terracol, A. (2002), "Analyzing the take-up of means-tested benefit in France", Working paper, París, Universidad de París.

Van Oorschot, W. (1996), "New perspectives on the non-take-up of social security benefits", TISSER Studies, Tilburg, Tilburg University Press.

(1991), "Non-take-up of social security benefits in Europe", Journal of European Social Policy, vol. 1, № 1, Thousand Oaks, Sage Publications. 